\title{
A Computational Modeling Account of Preference Reversal in Multi-attribute Decision Making
}

\author{
Feng Gao \\ School of Electric and Electronic Engineering, Wuhan Polytechnic University, 68 Xuefu South Road, Changqing Garden, Wuhan \\ 430023, Hubei, China
}

\begin{abstract}
In multi-attribute decision making, attribute conflict and indetermination preference of the decision maker often give rise to preference reversal, which is a marked discrepancy between the preferences derived from choice and from matching judgments, thus violating traditional decision theories. To account for such effect, a computational model of preference reversal in multi-attribute decision making is developed. The simulation results show that the model captures several consistent empirical findings.
\end{abstract}

Keywords-preference reversal; choice and matching; multiattribute decision making; dynamic decision models

\section{INTRODUCTION}

Preference can be inferred from direct choice between options or from a matching procedure in which the decision maker adjusts one option to match another. During the past 30 years of empirical research on the measurement of preference, researchers have found systematic preference reversal among these measurement methods. This preference reversal challenges traditional decision theories. The standard analysis of choice assumes procedure invariance: normatively equivalent procedures for assessing preferences should give rise to the same preference order. Indeed, theories of measurement generally require the ordering of objects to be independent of the particular method of assessment. To determine the heavier of two objects, for example, we can place them on the two sides of a pan balance and observe which side goes down. Alternatively, we can place each object separately on a sliding scale and observe the position at which the sliding scale is balanced. Similarly, to determine the preference order between options we can use either choice or matching. Note that the pan balance is analogous to binary choice, whereas the sliding scale resembles matching.

The discrepancy between choice and matching was first observed in a study by Slovic [1] that was motivated by the ancient philosophical puzzle of how to choose between equally attractive alternatives. Tversky, Sattath, and Slovic [2] develop the contingent weighting model which is applied to the analysis of various compatibility effects, including the choice-matching discrepancy and the preference reversal phenomenon. However, their model cannot explain the reversals between WTP (willing to pay) and WTA (willing to accept) and cannot explain choice-pricing reversals among gambles with equally probable outcomes. Johnson and Busemeyer [3] present a computational model that accounts for the empirical trends without changing decision weights, values, or combination rules. But the model cannot explain preference reversal in multi-attribute decision making. In this article, the author presents a new computational model based on decision field theory (DFT) [4] to account preference reversal in multi-attribute decision making.

\section{Tests of Preference ReVERSAL in Multi-AtTribute DECISION MAKING}

In this section we develop a method for testing the preference reversal phenomenon that is based entirely on interpersonal (between-subjects) comparisons, and we apply this method to a variety of choice problems. The subjects in the tests were undergraduates (ages 19-24 years) who were taking a series of aptitude tests. The problems were presented in writing, and the subjects were tested in small groups. They all agreed to take part in the study knowing it had no bearing on their test scores.

Test 1: The first group of subjects required to choose between A and B in Table 1 of options, each program contains two attributes: improving health quality (buy disinfection cabinet, ultraviolet light disinfection, etc.) and upgrading computer (purchasing cards, cpu, memory, etc.).

TABLE I. THE CHIOCE BETWEEN TWO OPTIONS

\begin{tabular}{|c|c|c|c|}
\hline Options & The cost of attributes 1 & The cost of attributes 2 & $\mathrm{~N}=30$ \\
\hline A & $¥ 1020$ & $¥ 900$ & $72 \%$ \\
\hline B & $¥ 920$ & $¥ 1080$ & $28 \%$ \\
\hline
\end{tabular}

The number of subjects $(\mathrm{N})$ and the percentage who chose each option are given in brackets on the right side of the table. In this problem, over two thirds of the subjects believe that improving health quality is more important, and they selected option A that has a higher cost on the attribute 1(buy disinfection cabinet, ultraviolet light disinfection, etc.).

Another group of subjects received the same data except that one of the four costs was missing. They were asked "to complete the missing cost so that the two options would be equally important." Suppose, for example, that the upper right cost (900) was missing from the table. The subject's task would then be to generate a cost for option A in upgrading computer so as to match the two options. The subjects were reminded that "A has a higher cost than B in improving health quality; hence, to match the two options A must have a lower cost than B in upgrading computer."

Assuming that higher costs are preferable to lower ones, it is possible to infer the response to the choice task from the 
response to the matching task. Suppose, for example, that one produces a value of 950 in the matching task. (when the missing value is 900). This means that B's cost profile (920, $1080)$ is judged equivalent to the profile $(1020,950)$, which in turn dominates A's profile $(1020,900)$. Thus, a matching value of 950 indicates that B is preferable to A. More generally, a matching response above 900 implies a preference for $\mathrm{B}$; a matching response below 900 implies a preference for $\mathrm{A}$; and a matching response of 900 implies indifference between $\mathrm{A}$ and B.

Let $\mathrm{n}_{\mathrm{C}}$ denote the percentage of subjects who chose $\mathrm{A}$ over $B$, and let $n_{M}$ denote the percentage of people whose matching response favored A over B. Assuming random allocation of subjects, procedure invariance implies $n_{C}=n_{M}$. To estimate $\mathrm{n}_{M}$, we presented four different groups of about 120 subjects each with the data of Test 1 , each with a different missing value, and we asked them to match the two options. The table 2 presents the values of $n_{M}$ derived from the matching data for each of the four missing values, which are given in parentheses.

TABLE II. THE PERCENTAGE OF PEOPLE WHOSE MATCHING RESPONSE FAVORED AOVER B

\begin{tabular}{|c|c|c|}
\hline Options & The cost of attributes 1 & The cost of attributes 2 \\
\hline A & $31 \%(1020)$ & $36 \%(900)$ \\
\hline B & $46 \%(920)$ & $28 \%(1080)$ \\
\hline
\end{tabular}

Test 2: Imagine that, as an executive of a company, you have to select between two candidates in Table 3 for a position of a Web Designer. The candidates were interviewed by a committee who scored them on two attributes (technical knowledge and human relations) on a scale from 100 (superb) to 30 (very weak). Both attributes are important for the position in question, but technical knowledge is more important than human relations. On the basis of the following scores, which of the two candidates would you choose?

TABLE III. THE CHIOCE BETWEEN TWO CANDIDATES

\begin{tabular}{|c|c|c|c|}
\hline Candidates & Technical knowledge & Human relations & $\mathrm{N}=30$ \\
\hline A & 88 & 78 & $67 \%$ \\
\hline B & 80 & 93 & $33 \%$ \\
\hline
\end{tabular}

As in Test 1 , most subjects $(67 \%, \mathrm{~N}=30)$ chose according to the more important attribute, but only $40 \%(\mathrm{~N}=120)$ of the matching responses favored A over B. Again, all four values of $\mathrm{n}_{\mathrm{M}}$ were smaller than $\mathrm{n}_{\mathrm{C}}$.

\section{COMPUTATIONAL Model BASE ON DFT}

DFT lies on the information processing theory and "approach-avoidance" theory of psychology. As a behavioral decision theory oriented about decision-making process, it dynamically approaches the cognition of human's decisionmaking process based on psychological rather than economic principles. DFT was initially applied to the study of decision making under uncertainty initially, and then to the research of decision-making behaviors such as multi-attribute decisions, multi-alternative choices, and multiple measures of preference
[5] [6]. To account for preference reversal in multi-attribute decision making, a computational model based on the decision field theory is developed. The computational model consists of multi-attribute dynamic choice model and multi-attribute dynamic matching model.

\section{A. Multi-attribute Dynamic Choice Model}

Figure 1 provides an interpretation of the Decision Field Theory as a connectionist network that has three layers [6]. The first layer computes the weighted utility according to the attributes of different options and attention weights as formulated in (1).

$$
(1) U_{i}(t)=\sum_{j} W_{j}(t) \cdot m_{i j}
$$

where, $U_{i}(t)$ is the weighted utility for option $i$ at time $t, m_{i j}$ is the evaluations for attribute $\mathrm{j}$ of option i. $W_{j}(t)$ is the momentary attention weights linked to attribute $j$. The average value of the attention weights corresponds to the weight in deterministic utility theory.

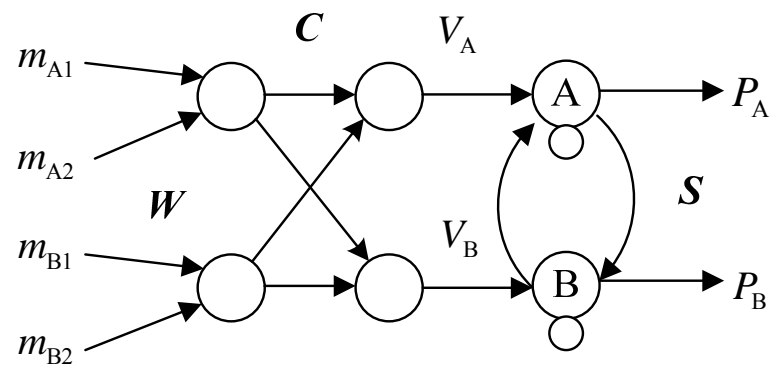

FIGURE I. CONNECTIONIST NETWORK MODEL OF DFT

The outputs of the second layer are valences which represent the advantage or disadvantage being considered for each option at a particular time point. These valences change stochastically over time as the decision maker's attention shifts unpredictably from one attribute to another.

$$
\begin{gathered}
v_{i}(t)=U_{i}(t)-U_{g}(t) \\
U_{g}(t)=\sum_{k \neq i} U_{k}(t) /(n-1)
\end{gathered}
$$

where, $v_{i}(t)$ is the valence for option i. $v_{i}(t)>0$ indicates that the option $i$ has an advantage under the current focus of attention while $v_{i}(t)<0$ indicates that the option $i$ has a disadvantage under the current focus of attention. $U_{g}(t)$ is the average utility of the other (n-1) options and $n$ is the number of options.

The third layer is a competitive recursive network. The outputs of this layer are the evolving accumulative preferences 
for the options at a particular time point. The accumulative preference is formed by the integration of the preference at previous time points and the temporal input valences. The preference state for option $\mathrm{i}$ is computed according to the linear dynamic system.

$$
\tilde{P}(t+h)=S \cdot \tilde{P}(t)+V(t+h)
$$

$\tilde{P}(t+h)$ (n-elements vector) represents a preference state for all options at time $t+h, V(t+h)$ is the valence vector for all options at time $t+h$, and $h$ is a time step. The feedback matrix $S$ of (4) represents the effect of the preference from the previous state (the memory effect) in the diagonal elements and the effect of the interactions among the options in the offdiagonal elements. For the stability of this linear system, the eigenvalues $\lambda_{i}$ of $S$ are assumed to be less than one in magnitude $\left(\left|\lambda_{i}\right|<1 \mid\right)$.Equation (4) can be further expanded as

$$
\tilde{P}_{i}(t+h)=s_{i i} \cdot \tilde{P}_{i}(t)+\sum_{k \neq i} s_{i k} \cdot \tilde{P}_{k}(t)+v_{i}(t+h)
$$

where $\tilde{P}_{i}(t+h)$ is the preference state for option $\mathrm{i}$ at time $\mathrm{t}+\mathrm{h}$. Equation (5) can be used for the comparison module of multiattribute dynamic matching model.

The stopping of decision process is controlled by threshold $\theta$ : Set a threshold $\theta$. If $\tilde{P}_{m}(t) \geq \theta, \tilde{P}_{j}(t)<\theta$, $j \neq m$, alternative $\tilde{P}_{m}(t)$, the first one to reach the threshold, is chosen. It worth mentioning that a decision-maker threshold bound is not fixed but rather varies according to the choice situation and time constraints.

\section{B. Multi-attribute Dynamic Matching Model}

Multi-attribute dynamic matching model consists of comparison module and matching module. Formally, let $\left(m_{\mathrm{A} 1}, m_{\mathrm{A} 2}\right)$ and $\left(m_{\mathrm{B} 1}, m_{\mathrm{B} 2}\right)$ denote the values of options $\mathrm{A}$ and $\mathrm{B}$ on attributes 1 and 2 . We use the subscript 1 to denote the primary, or the more important dimension, and the subscript 2 to denote the secondary, or the less important dimension- whenever they are defined. If neither option dominates the other, A denotes the option that is superior on the primary dimension and $\mathrm{B}$ denotes the option that is superior on the secondary dimension, thus, $m_{\mathrm{A} 1}>m_{\mathrm{B} 1}, m_{\mathrm{B} 2}>m_{\mathrm{A} 2}$.

The comparison module is used to compare options $\mathrm{A}$ and $\mathrm{B}$, and comparison results can be obtained directly from (5). If $\tilde{P}_{\mathrm{A}}(t) \geq \theta, \tilde{P}_{\mathrm{B}}(t)<\theta$, option $\mathrm{A}$ is chosen. If $\tilde{P}_{\mathrm{B}}(t) \geq \theta$, $\tilde{P}_{\mathrm{A}}(t)<\theta$, option B is chosen. If $\tilde{P}_{\mathrm{A}}\left(t^{*}\right)=\tilde{P}_{\mathrm{B}}\left(t^{*}\right)\left(t^{*} \neq 0\right)$, options $\mathrm{A}$ and $\mathrm{B}$ is indifference.

The matching module is used to search for a value $m^{*}$ that causes indifference when paired with other options from a set $m=\left[\begin{array}{llll}m_{1} & m_{2} & \cdots & m_{l}\end{array}\right]$ of 1 candidate attribute values, in which $m_{l}>\cdots>m_{2}>m_{1}$, and $m^{*}=\tilde{m}_{i j}, \tilde{m}_{i j}$ is missing value of option $i$ on attribute $j$. Specifically, by selecting a step size $\Delta_{\mathrm{M}}$ for the matching process, we obtain $\Delta_{\mathrm{M}}=\left(m_{l}-m_{1}\right) / l$. By assumption of $m_{\mathrm{A} 1}>m_{\mathrm{B} 1}$, $m_{\mathrm{B} 2}>m_{\mathrm{A} 2}$, the distribution of options $\mathrm{A}$ and $\mathrm{B}$ on candidate attribute values is $\tilde{m}_{\mathrm{A} 1} \in\left(m_{\mathrm{B} 1}, \infty\right), \tilde{m}_{\mathrm{A} 2} \in\left[0, m_{\mathrm{B} 2}\right)$, $\tilde{m}_{\mathrm{B} 1} \in\left[0, m_{\mathrm{A} 1}\right), \tilde{m}_{\mathrm{B} 2} \in\left(m_{\mathrm{A} 2}, \infty\right)$.

For example, $\tilde{m}_{\mathrm{A} 2}$ is missing attribute value of option $\mathrm{A}$ on attribute 2. Assuming that higher attribute values are preferable to lower ones, it is possible to search an attribute value for option A so as to match options A and B. The matching module is applied to adjust a candidate attribute value up and down in search of indifference and described as

$$
\begin{array}{cc}
m_{i-1}(n+1)=m_{i}(n)-\Delta_{\mathrm{M}} & \tilde{P}_{\mathrm{A}}(t) \geq \theta, \tilde{P}_{\mathrm{B}}(t)<\theta \\
m_{i}(n+1)=m_{i-1}(n)+\Delta_{\mathrm{M}} & \tilde{P}_{\mathrm{B}}(t) \geq \theta, \tilde{P}_{\mathrm{A}}(t)<\theta \\
\tilde{m}_{\mathrm{A} 2}=m_{i}(n) & \tilde{P}_{\mathrm{A}}\left(t^{*}\right)=\tilde{P}_{\mathrm{B}}\left(t^{*}\right)
\end{array}
$$

These equations simply state that the candidate value is either incremented or decremented by some constant amount $\Delta_{M}$ from one comparison (n) to the next $(\mathrm{n}+1)$ unless $\tilde{P}_{\mathrm{A}}\left(t^{*}\right)=\tilde{P}_{\mathrm{B}}\left(t^{*}\right)$, in which case the matching process ends and reports $\tilde{m}_{\mathrm{A} 2}=m_{i}(n)$. Each comparison is determined by the comparison module.

Suppose $\tilde{m}_{\mathrm{A} 2}>m_{\mathrm{A} 2}$, then $\left(m_{\mathrm{A} 1}, \tilde{m}_{\mathrm{A} 2}\right)$ is equivalent to $\left(m_{\mathrm{B} 1}, m_{\mathrm{B} 2}\right)$ by matching, $\left(m_{\mathrm{A} 1}, \tilde{m}_{\mathrm{A} 2}\right)$ is preferred to $\left(m_{\mathrm{A} 1}, m_{\mathrm{A} 2}\right)$ by dominance; hence, $\mathrm{B}$ is preferred to $\mathrm{A}$ by transitivity. The other cases are similar.

\section{Quantitative AnAlysis of PrefEREnCE REVERsal}

The data described in Section II show a marked discrepancy between choice and matching. Section III presents a new computational model that consists of multi-attribute dynamic choice model and matching model. To apply the computational model to accounts for the preference reversal phenomenon, algorithm implemented within Matlab is shown in Fig. 2.

For example, $\widetilde{m}_{\mathrm{A} 2}$ is missing attribute value of option A on attribute 2. Repeat multi-attribute dynamic matching model for $\mathrm{N}$ cycles $(\mathrm{N} \geq 1000)$. Suppose $\tilde{P}_{\mathrm{A}}\left(t^{*}\right)=\tilde{P}_{\mathrm{B}}\left(t^{*}\right)$ is selected 
for $\left[\begin{array}{llll}\mathrm{N}_{1} & \mathrm{~N}_{2} & \cdots & \mathrm{N}_{l}\end{array}\right]$ times when corresponding candidate attribute value is $\left[\begin{array}{llll}m_{1} & m_{2} & \cdots & m_{l}\end{array}\right]$, then the resulting indifference probabilities for each candidate attribute value is $\left[\begin{array}{lllll}\mathrm{N}_{1} / \sum_{i=1}^{l} \mathrm{~N}_{i} & \mathrm{~N}_{2} / \sum_{i=1}^{l} \mathrm{~N}_{i} & \cdots & \mathrm{N}_{l} / \sum_{i=1}^{l} \mathrm{~N}_{i}\end{array}\right]$. As mentioned in Section II, when $\tilde{m}_{\mathrm{A} 2}<m_{\mathrm{A} 2}$, option A is chosen; $\tilde{m}_{\mathrm{A} 2}>m_{\mathrm{A} 2}$, option $\mathrm{B}$ is chosen; $\tilde{m}_{\mathrm{A} 2}=m_{\mathrm{A} 2}$, options

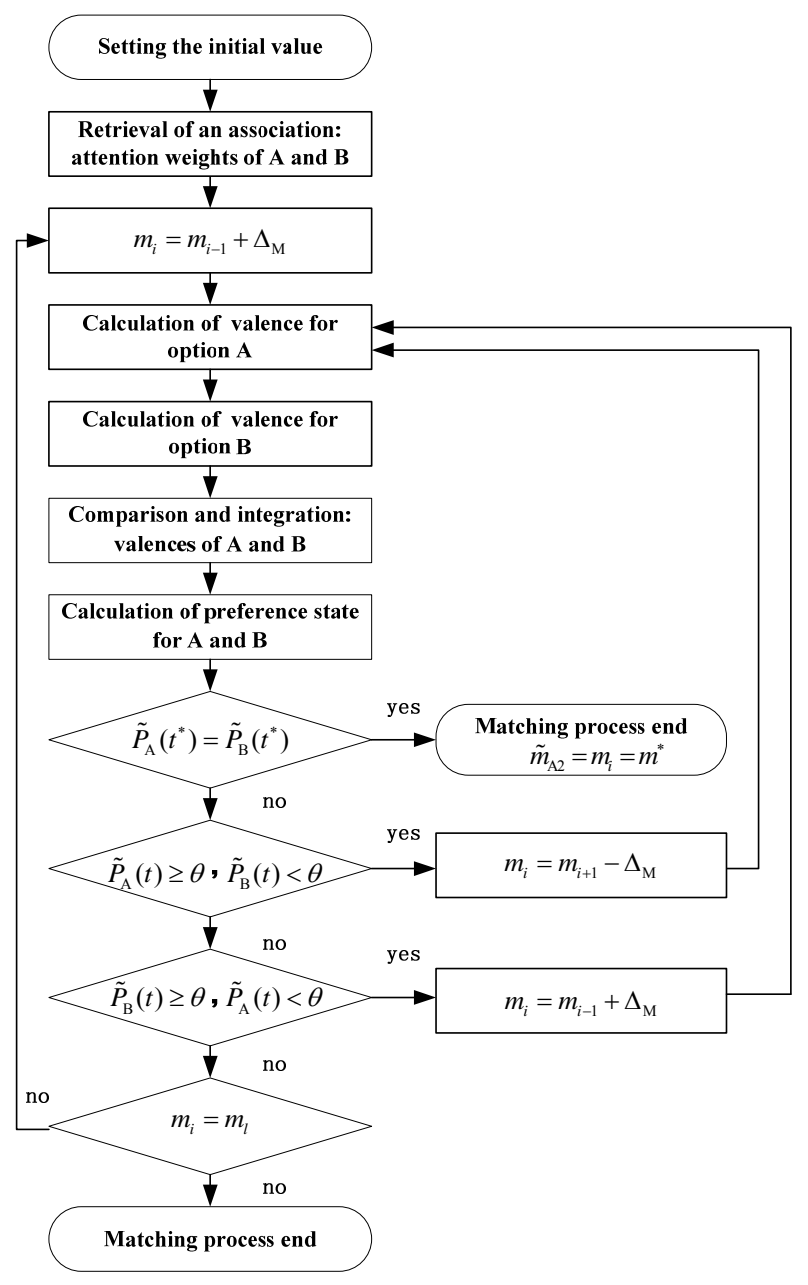

FIGURE II. ILLUSTRATION OF ALGORITHM

TABLE IV. CHOICE PROBABILITIES OF THE MODEL IN 2 SIMULATED TEST (CHOOSING OPTION A)

\begin{tabular}{|c|c|c|c|c|c|}
\hline $\begin{array}{c}\text { Measurement } \\
\text { Comparison }\end{array}$ & Choice & \multicolumn{4}{|c|}{ Matching } \\
\hline & & $31 \%$ & $36 \%$ & $46 \%$ & $28 \%$ \\
Test 1 & $72 \%$ & $(1020)$ & $(900)$ & $(920)$ & $(1080)$ \\
Model & $68 \%$ & $35 \%$ & $32 \%$ & $39 \%$ & $31 \%$ \\
& & $(1020)$ & $(900)$ & $(920)$ & $(1080)$ \\
\hline & & $33 \%$ & $31 \%$ & $46 \%$ & $28 \%$ \\
Test 2 & $67 \%$ & $(88)$ & $(78)$ & $(80)$ & $(93)$ \\
Model & $65 \%$ & $35 \%$ & $28 \%$ & $44 \%$ & $25 \%$ \\
& & $(88)$ & $(78)$ & $(80)$ & $(93)$ \\
\hline
\end{tabular}

$\mathrm{A}$ and $\mathrm{B}$ is indifference. It is possible to infer the probability of choosing options $\mathrm{A}$ or $\mathrm{B}$, and the probability of an indifference response between options $\mathrm{A}$ and $\mathrm{B}$. The simulation results in Table 4 show that the model captures several consistent empirical findings.

\section{CONCLUSION}

In this paper, the quantitative description of preference reversal is shown and the construction of preference is analyzed on psychological tests. To account for the marked discrepancy between the preferences derived from choice and from matching judgments, a computational model based on DFT is developed, which consists of multi-attribute dynamic choice model and matching model. To apply the computational model to accounts for the preference reversal phenomenon in multi-attribute decision making, algorithm implemented within Matlab is developed. The simulation results show that the model captures several consistent empirical findings.

\section{ACKNOWLEDGMENT}

This research is supported by the National Natural Science Foundation of China under Grant No. 60274047, and Grant No. 60874068 .

\section{REFERENCES}

[1] Slovic, P., "Choice between equally valued alternatives," Journal of Experimental Psychology: Human Perception and Performance, vol.1, pp.280-287, 1975 .

[2] Tversky,A., Sattath,S. , and Slovic,P., "Contingent Weighting in Judgment and Choice," Psychology Review, vol.95, pp.371-384, July 1988

[3] Johnson J. G. and Busemeyer, J. R. , "A dynamic, stochastic, computational model of preference reversal phenomena," Psychological Review, vol. 112, pp. 841-861,October 2005

[4] Busemeyer,J. R. and Townsend, J.T., "Decision field theory: A dynamic-cognitive approach to decision making in an uncertain environment," Psychological Review, vol. 100, pp. 432-459, July 1993

[5] Johnson, J.G. and Busemeyer , J.R., "Decision making under risk and uncertainty," WIREs Cognitive Science, vol. 1, pp. 736-749, September 2010

[6] Johnson, J.G. and Busemeyer, J.R., "A computational model of the attention process in risky choice," Decision, 3 (4), pp.254-280. 2016

[7] Busemeyer, J. R., Jessup, R. K., and Dimperio, E., "The dynamic interactions between situations and decisions," In P. Robbins, M. Aydede (Eds.), The Cambridge Handbook of Situation Cognition, Cambridge University Press, New York, 2009, pp. 307-321. 